\title{
Vulnerability profile of migrant construction workers to HIV/AIDS in an urban area in south India - a cross sectional study
}

\author{
Ajoke Basirat Akinola ${ }^{1}$, Anil Kumar Indira Krishna ${ }^{2}$ Satish Kumar Chetlapalli ${ }^{3}$ \\ ${ }^{\prime}$ (School of Public health, Sri Ramaswamy Memorial University, Tamil Nadu, India) \\ ${ }^{2}$ (School of Public health, Sri Ramaswamy Memorial University, Tamil Nadu, India) \\ ${ }^{3}$ (School of Public health, Sri Ramaswamy Memorial University, Tamil Nadu, India)
}

\begin{abstract}
Introduction: It is well known that migrant construction workers are at high risk for HIV/AIDS. Their vulnerability profile is not well known. Objectives: To assess the vulnerability profile of migrant construction workers to HIV/AIDS in an urban area in south India. Methods: A cross sectional descriptive assessment was done among 307 migrant construction workers from 15 constructions sites in an urban area in Chennai selected through multi stage random sampling using a structured questionnaire. Results: Nearly $85 \%$ of the respondents were aged below 35 years, 92\% were living alone, $76 \%$ lacked education, and more than $60 \%$ had migrated greater than 5 years ago. Other than HIV being spread by sharing needles, they had a good level of knowledge and awareness about transmission of HIV. But they were not aware of Anti-retroviral treatment. There were no misconceptions among the migrant workers. Conclusions: Though levels of knowledge and awareness about HIV/AIDS are high among the migrant workers, the vulnerability levels are very high. There needs to be vulnerability mitigating interventions at the policy level apart from increasing awareness.
\end{abstract}

Keywords: AIDS, HIV, knowledge, migrant worker, vulnerability

\section{INTRODUCTION}

India is currently facing the phenomenon of heavy internal migration. The construction industry is the major employer of these internally migrant populations. The number of migrant construction workers has increased from 40 million in 2008 to 258 million currently. [1-3] India is also the home for the third largest number of persons living with HIV/AIDS in the world with close to 2.5 million people currently living with HIV/AIDS. [1] There are several special population groups in India who are at high risk for HIV/AIDS and migrant workers are one of them. [1] Vulnerability has been defined in several ways, medical, epidemiological, social and political.[4] The migrant population is unique in that they are vulnerable in all these ways. [4] This study was done to understand the vulnerability profile of migrant construction workers to HIV/AIDS in an urban area in south India.

\section{Methodology}

The study was carried out using a cross sectional design in a semi urban area of south India. A structured questionnaire was designed to capture information on socio-demographic characteristics, migration patterns, vulnerability factors and knowledge about HIV/AIDS. Sample size was calculated for a $95 \%$ confidence limit, $10 \%$ relative precision of estimate and $50 \%$ assumed prevalence of high vulnerability. The required sample size was 300 . Fifteen construction sites were selected in the urban and semi-urban areas of Chennai by random sampling method. The number of respondents from each site was selected by population proportion method. The respondents were selected by simple random sampling within each site from the list available with the person in charge of the site. The information was gathered through face to face interviews at the construction site after obtaining written informed consent. The data were entered in Epi Info software version 3.5.1 and analysis was done using Statistical Package for Social Sciences (SPSS) version 17. Simple descriptive statistics were calculated to understand the prevalence and profile of each factor inducing vulnerability. The study protocol was reviewed by the Ethical Committee of the School of Public Health, SRM University and approved.

\section{RESULTS}

A total of 307 interviews were conducted in the 15 construction sites. All contacted individuals consented and responded to the questionnaires. The characteristics of the respondents are shown in table 1 . Nearly $85 \%$ of the respondents were aged below 35 years, $92 \%$ were living alone, $76 \%$ lacked education, and 
more than $60 \%$ had migrated greater than 5 years ago. The other vulnerability factors were much lesser in the sample. The prevalence of each of these factors of vulnerability are shown in Table 2 . Table 3 depicts the level of knowledge about each of the domains of transmission, testing and treatment of HIV/AIDS. It also depicts information about the misconceptions and wrong beliefs about HIV/AIDS. Most of the respondents had good knowledge about HIV/AIDS.

\section{DISCUSSION}

This study reveals that there is a high level of vulnerability among the migrant workers working in Chennai with respect to HIV/AIDS. Several studies have shown that young age is a high vulnerability factor in the transmission of HIV due to the high level of sexual activity which is often indiscriminate and unprotected. [5] But youth is also the economically productive time when migration happens most. This leads to high level of vulnerability of the young migrant workers. Studies have also shown that living alone leads to a higher risk of vulnerability to HIV transmission.[6] This study also showed a high proportion of migrant workers who were living alone. Lack of education and long duration of migration have also been shown to have higher risk for HIV. The study revealed that vulnerability in terms of high risk sexual behavior was low in the study population. But the results have to be interpreted with caution as there is a possibility of non-reporting or underreporting of sexual behaviors in the sample.

Knowledge levels about HIV/AIDS were also found to be very low among the sampled construction workers. While the workers knew that infected blood or organs, sexual intercourse with infected person and transmission from infected mother to child can happen, they did not know that sharing needles with infected individuals will lead to transmission of the disease. This puts them at a high vulnerability for injectable drug use related HIV transmission. There was poor awareness about anti- retroviral therapy among the migrant workers. But they seemed to have correct understanding about the disease and did not have much misconception. These findings reflect the high level of awareness generated by information, education, communication campaigns about HIV/AIDS in the community.

The findings of the study reveal that there is distinctly high vulnerability level among migrant construction workers. But the level of knowledge and beliefs about HIV seem to be high. This is a positive finding for program planner and policy makers. In future interventions should be planned to mitigate the factors leading to high vulnerability. It is not sufficient to just increase knowledge and awareness. Some of the vulnerability mitigating interventions are: ensuring group accommodations for migrant workers and adequate peer support to prevent risky sexual behaviors, registration of migrant workers to ensure that all workers are of employable age and minimum educational attainment. The findings of this study can be used to develop further studies in this area of vulnerability. A valid vulnerability index can be developed which can be used to screen migrant workers for high risk and appropriate interventions provided.

\section{FIGURES AND TABLES}

\begin{tabular}{lll}
\hline Indicators & Frequency & Percentage \\
Age & & 3.3 \\
$<18$ & 10 & 82.4 \\
$18-34$ & 253 & 14.3 \\
$>35$ & 44 & 82.7 \\
Religion & 254 & 14.7 \\
Hindu & 45 & 1.6 \\
Muslim & 5 & 0.3 \\
Christian & 1 & 0.7 \\
None & 2 & \\
$\quad$ Others & 16 & 5.2 \\
Caste & 46 & 15.0 \\
MBC & 104 & 33.9 \\
OBC & 14 & 4.6 \\
SC & 127 & 41.4 \\
ST & & \\
$\quad$ Others & 108 & 35.2 \\
Marital status & 199 & 68.4 \\
Married & & \\
Unmarried (Single, Divorced, Separated) & 235 & 76.5 \\
Education & 72 & 23.5 \\
Without education (no education, can read and write simple massage) & \\
With Education(primary, high school, secondary, others) & & \\
\hline
\end{tabular}

Table 1: Socio demographic characteristics of migrant construction workers 
Table 2: Prevalence of vulnerability factors among migrant construction workers $(n=307)$

\begin{tabular}{|c|c|c|}
\hline Vulnerability Factor & Frequency & Percentage \\
\hline Age $<35$ years & 263 & $85.7 \%$ \\
\hline Living alone & 284 & $92.5 \%$ \\
\hline Self arranged accommodation & 7 & $2.3 \%$ \\
\hline Did not have any education & 235 & $76.5 \%$ \\
\hline Early age at migration $(<18$ years $)$ & 172 & $56 \%$ \\
\hline Long duration of migration ( $>5 \mathrm{yrs}$ ) & 189 & $61.6 \%$ \\
\hline Multiple sexual partners & 13 & $4.2 \%$ \\
\hline Contact with paid sex workers & 7 & $2.3 \%$ \\
\hline Not using condoms regularly & 3 & $1 \%$ \\
\hline
\end{tabular}

Table 3: Knowledge, misconceptions and beliefs about HIV/AIDS among migrant workers

\begin{tabular}{|c|c|}
\hline Knowledge and Misconception of HIV/AIDS & No \\
\hline $\begin{array}{l}\text { Transmission: Sharing needles with HIV - infected person? } \\
\mathrm{N}=181\end{array}$ & 88.4 \\
\hline $\begin{array}{l}\text { Transfusion of HIV-infected blood or receiving HIV-infected organ? } \\
\mathrm{N}=201\end{array}$ & 13.4 \\
\hline $\begin{array}{l}\text { From an HIV-positive mother to her fetus? } \\
\mathrm{N}=176\end{array}$ & 12.5 \\
\hline $\begin{array}{l}\text { Through sexual intercourse? } \\
\mathrm{N}=197\end{array}$ & 4.1 \\
\hline $\begin{array}{l}\text { Treatment: There is vaccine for AIDS. } \\
\mathrm{N}=300\end{array}$ & 48.0 \\
\hline $\begin{array}{l}\text { Antiretroviral drugs that people infected with HIV/AIDS can get from a doctor or } \\
\text { nurse to help them live longer? } \\
\mathrm{N}=61\end{array}$ & 49.1 \\
\hline $\begin{array}{l}\text { Spreads through mosquito bites? } \\
\mathrm{N}=152\end{array}$ & 93.8 \\
\hline $\begin{array}{l}\text { Sharing a meal with an HIV-infected person? } \\
\mathrm{N}=114\end{array}$ & 75.5 \\
\hline $\begin{array}{l}\text { Sharing personal items such as shaving blades? } \\
\mathrm{N}=194\end{array}$ & 24.4 \\
\hline $\begin{array}{l}\text { Bathing in a stream or public toilet? } \\
\mathrm{N}=184\end{array}$ & 80.3 \\
\hline $\begin{array}{l}\text { Contacts such as hugging or touching with an HIV-infected person } \\
N=197\end{array}$ & 81.8 \\
\hline $\begin{array}{l}\text { The appearance of HIV carriers is different from normal population? } \\
\mathrm{N}=178\end{array}$ & 37.1 \\
\hline
\end{tabular}
$\mathrm{N}=178$

\section{Conclusion}

In an urban area in south India, the level of vulnerability regarding HIV/AIDS is very high, but there is adequate awareness and knowledge about the disease. There is a need to implement vulnerability mitigating measures at the policy level apart from increasing knowledge and awareness.
[1] UNAIDS,2010-
India
https://www.unaids.org/en/dataanalysis/knowyourresponse/countryprogressreports/2010countries/india_2010_country progress_re port_en.pdf
[2] Niranjan Saggurti et al., Technical briefing from population council India. Pattern and Implication of male migration for HIV prevention strategies in Karnataka, India, 2008. www.popcouncil.org/pdfs/India_TechBriefKarnataka.pdf
[3] Deshingkar, Priya, and ShaheenAkter. Migration and human development in India. (2009).
[4] Wolffers, Ivan, et al. Sexual behaviour and vulnerability of migrant workers for HIV infection. Culture, health \& sexuality 4.4 (2002): 459-473.
[5] Puri M, Cleland J.Sexual behavior and perceived risk of HIV/AIDS among young migrant factory workers in Nepal. $J$ Adolesc Health. 2006 Mar;38(3):237-46.
[6] Rwenge M. Sexual Risk Behaviors Among Young People in Bamenda, Cameroon. IntFam Plan Persp 2000;26(3):118-23.

REFERENCES 